\title{
Neural Processing of Risk
}

\author{
Peter N. C. Mohr, ${ }^{1,2,3}$ Guido Biele, ${ }^{1,2,3}$ and Hauke R. Heekeren ${ }^{1,2,3}$ \\ ${ }^{1}$ Max Planck Institute for Human Development, D-14195 Berlin, Germany, ${ }^{2}$ Max Planck Institute for Human Cognitive and Brain Sciences, D-04103 Leipzig, \\ Germany, and ${ }^{3}$ Freie Universität Berlin, D-14195 Berlin, Germany
}

In our everyday life, we often have to make decisions with risky consequences, such as choosing a restaurant for dinner or choosing a form of retirement saving. To date, however, little is known about how the brain processes risk. Recent conceptualizations of risky decision making highlight that it is generally associated with emotions but do not specify how emotions are implicated in risk processing. Moreover, little is known about risk processing in non-choice situations and how potential losses influence risk processing. Here we used quantitative meta-analyses of functional magnetic resonance imaging experiments on risk processing in the brain to investigate (1) how risk processing is influenced by emotions, (2) how it differs between choice and non-choice situations, and (3) how it changes when losses are possible. By showing that, over a range of experiments and paradigms, risk is consistently represented in the anterior insula, a brain region known to process aversive emotions such as anxiety, disappointment, or regret, we provide evidence that risk processing is influenced by emotions. Furthermore, our results show risk-related activity in the dorsolateral prefrontal cortex and the parietal cortex in choice situations but not in situations in which no choice is involved or a choice has already been made. The anterior insula was predominantly active in the presence of potential losses, indicating that potential losses modulate risk processing.

\section{Introduction}

Many decisions in our everyday life, such as choosing a restaurant for dinner or choosing a form of retirement saving, can be described as decisions under risk. Decision sciences such as psychology and economics usually define risk as the uncertainty about which of several possible outcomes will occur, whereby the probability of each possible outcome is known (Knight, 1921). In contrast, ambiguity describes a form of uncertainty in which probabilities and/or possible outcomes are unknown.

Traditional models of risky decision making implicitly assume that the characteristics of a choice option (e.g., magnitude of possible outcomes) are cognitively evaluated. In the framework of expected utility theory, people's willingness to take risk depends on the concavity of the utility function. In prospect theory, it additionally depends on the shape of the probability weighting function (for review, see Bossaerts et al., 2009; Fox and Poldrack, 2009).

Recent approaches, however, highlighted the role of emotions in decision making. Based on psychological and neuroscientific research, theories such as the affect heuristic (Slovic et al., 2004), the risk-as-feelings hypothesis (Loewenstein et al., 2001), and the somatic marker hypothesis (Damasio et al., 1991) propose that emotions interact with a cognitive evaluation of the choice problem to guide behavior. To date, however, it remains unclear how risk processing is influenced by emotions.

Received Jan. 1, 2010; revised March 16, 2010; accepted March 29, 2010.

This research was supported by the Max Planck Society and by the German Research Foundation (Cluster of Excellence "Languages of Emotion," EXC 302). P.N.C.M. was supported by a predoctoral fellowship of the International Max Planck Research School (The Life Course: Evolutionary and Ontogenetic Dynamics). We thank Angela Laird for helpful comments on the setup of the ALE meta-analyses.

Correspondence should be addressed to Peter N.C. Mohr, Neurocognition of Decision Making Group, Max Planck Institute for Human Development, Lentzeallee 94, 14195 Berlin, Germany. E-mail: mohr@mpib-berlin.mpg.de.

DOI:10.1523/JNEUROSCI.0003-10.2010

Copyright $\odot 2010$ the authors $\quad 0270-6474 / 10 / 306613-07 \$ 15.00 / 0$
Traditional models of risky decision making further often assume that risk is a context-independent function of the variability of possible outcomes (e.g., variance) (Markowitz, 1952). Research on the perception of risk, however, indicates that risk perception is neither context independent nor a pure measure of outcome variability (Weber et al., 2002). One can distinguish risk processing during or before choice (decision risk) and risk processing after or without a choice (anticipation risk), with the crucial difference that risk information is likely used to guide choices in the context of decision risk but not in the context of anticipation risk. Furthermore, individuals' judgments of perceived risk are more sensitive to downside variability and losses than to upside variability, indicating that risk might be processed differently if losses are possible (Weber et al., 1992).

Thus, three main questions regarding risk processing remain unresolved, namely (1) how risk processing is generally influenced by emotions, (2) whether the neural processing of risk is context dependent, differing between decision making and pure outcome anticipation, and (3) whether risk is processed differently in the brain when individuals are faced with potential losses.

Because single studies use specific tasks, their ability to answer such general questions is limited. In contrast, quantitative metaanalyses provide unbiased, objective measures of brain functioning and provide a useful approach to address such questions. Here, we performed quantitative voxelwise meta-analyses on neural representations of risk using the activation likelihood estimation method (ALE) (Turkeltaub et al., 2002; Laird et al., 2005). Importantly, ALE also allows a comparison of different task conditions (e.g., decision and anticipation) that were not contrasted in the same study.

\section{Materials and Methods}

Study selection. To identify studies investigating the neural processing of risk, we performed a literature search in two databases, namely Web of 
Science and Medline. Both databases were searched with the following logic conjunction of keywords: ("risk" OR "uncertainty") AND ("decision making" OR "choice" OR "anticipation") AND ("fMRI” OR "neuroimaging"). The search (performed on November 1, 2009) identified 285 hits. We further identified recent review papers about risky decision making that explicitly discuss the issue of risk processing in the brain (Knutson and Bossaerts, 2007; Platt and Huettel, 2008; Rangel et al., 2008; Rushworth and Behrens, 2008; Mohr et al., 2010b). All studies found through the database search and those that were cited by one of these review papers underwent the study selection process. The full selection process included a reading of introduction and methods part of each article by at least one of the three authors, followed by application of the following inclusion criteria: (1) functional magnetic resonance imaging (fMRI) study involving healthy young adult human participants; (2) imaging data acquired over the whole brain; (3) availability of peak activation coordinates from group activation maps; (4) outcomes of the task are at least partly uncertain; (5) available information for participants regarding outcome probabilities; and (6) outcomes of the task are independent of the behavior of others.

The inclusion criteria were chosen to ensure that our results could be generalized to the population of young healthy adult humans. Several studies on cognitive aging showed that cognitive changes across the adult lifespan are paralleled by structural and functional changes in the brain (Cabeza et al., 2005; Mohr et al., 2010b). Furthermore, older adults often show activations that are qualitatively different from those of young adults (Park et al., 2004). Therefore we included only studies that investigated risk processing in young healthy adults (criterion 1 ).

Driven by specific functional hypotheses, some neuroimaging studies do not acquire images of the whole brain but only of parts of the brain, allowing a higher image resolution. These images, however, impede the detection of nonhypothesized activations in brain regions that were not scanned. That is why criterion 2 excludes studies that did not scan the whole brain.

Because the ALE approach is based on activation foci (see below), we only included studies that report peak activation coordinates of group activation maps (criterion 3).

Because risk is usually contrasted with ambiguity-a form of uncertainty in which probabilities are unknown - we included only studies in which outcomes are uncertain (criterion 4) and probabilities are known, learned, or could be estimated (criterion 5). We further limited the included studies/analyses to those in which outcomes are independent of the behavior of others (criterion 6), because these might be confounded with other effects of, e.g., trust or reciprocity.

Because of non-independence considerations, we set an additional inclusion criterion on the level of analyses. If two risk analyses (e.g., contrast and correlation) were performed on the same dataset (and same time window), we included only the more specific risk analysis (correlation) because it reflects risk processing likely better than the less specific analysis (contrast).

Thirty studies met the inclusion criteria (Table 1) (supplemental Table S1, available online on www.jneurosci.org), representing 232 risk-related foci. Fifteen studies (101 foci) compared conditions, whereas 15 studies (131 foci) correlated risk with brain activity. Twenty-one studies (172 foci) investigated decision risk, and 10 studies (60 foci) investigated anticipation risk (one study investigated both). We further categorized studies according to potential outcomes. Eleven studies (101 foci) investigated risk in the context of only gains, whereas in 11 (92 foci) studies gains and losses were possible outcomes. For two studies ( 28 foci), the categorization was ambiguous because they investigated objective gains but included an obvious reference point that could lead to the perception of some positive outcomes as losses (with regard to the reference point). The tasks of six studies (39 foci) did not include monetary gains or losses (e.g., only right/wrong as outcomes).

ALE meta-analysis. In contrast to meta-analyses in behavioral sciences that aim to estimate the true effect size for an effect, fMRI meta-analyses aim to identify networks of brain regions implicated in certain cognitive processes (Turkeltaub et al., 2002). The focal question of fMRI metaanalyses is thus about the location of brain activity associated with specific cognitive processes rather than about the effect size of the
Table 1. Included risk studies

\begin{tabular}{|c|c|c|c|c|c|c|c|c|}
\hline & \multirow{2}{*}{$\begin{array}{l}\text { No. of } \\
\text { risk-related foci }\end{array}$} & \multicolumn{2}{|l|}{ Context } & \multicolumn{2}{|c|}{ Analysis } & \multicolumn{3}{|c|}{ Domain } \\
\hline & & $\overline{D R}$ & AR & Corr & Contr & $\bar{G}$ & $G+\mathrm{L}$ & 0 \\
\hline Volz et al. (2004) & 5 & $\mathrm{x}$ & & $\mathrm{x}$ & & $\mathrm{x}$ & & \\
\hline Volz et al. (2003) & 7 & $\mathrm{x}$ & & $\mathrm{x}$ & & $\mathrm{x}$ & & \\
\hline Preuschoff et al. (2006) & 22 & & $\mathrm{x}$ & $\mathrm{x}$ & & & $x$ & \\
\hline Paulus et al. (2003) & 5 & $x$ & & & $\mathrm{x}$ & & $x$ & \\
\hline Paulus et al. (2001) & 10 & $\mathrm{x}$ & & & $\mathrm{x}$ & $\mathrm{x}$ & & \\
\hline Preuschoff et al. (2008) & 16 & & $\mathrm{x}$ & $\mathrm{x}$ & & & $x$ & \\
\hline Critchley et al. (2001) & 4 & & $\mathrm{x}$ & $\mathrm{x}$ & & & $x$ & \\
\hline Kuhnen and Knutson (2005) & 2 & & $\mathrm{x}$ & $x$ & & & $x$ & \\
\hline Matthews et al. (2004) & 4 & $x$ & & & $x$ & & $x$ & \\
\hline Tobler et al. (2006) & 1 & & $\mathrm{x}$ & & $x$ & $x$ & & \\
\hline Huettel et al. (2005) & 10 & $x$ & & $x$ & & & & $x$ \\
\hline Hsu et al. (2005) & 12 & $x$ & & & $x$ & & & $x$ \\
\hline Dreher et al. (2006) & 4 & & $\mathrm{x}$ & & $x$ & & $x$ & \\
\hline Huettel (2006) & 15 & $x$ & & $x$ & & $x$ & & \\
\hline Grinband et al. (2006) & 8 & $x$ & & $x$ & & & & $x$ \\
\hline Behrens et al. (2007) & 1 & & $\mathrm{x}$ & $x$ & & $x$ & & \\
\hline Rolls et al. (2008) & 1 & & $\mathrm{x}$ & $x$ & & $x$ & & \\
\hline Yoshida and Ishii (2006) & 3 & $x$ & & $x$ & & & & $x$ \\
\hline Smith et al. (2009) & 2 & $x$ & & & $x$ & $x$ & & \\
\hline Weber and Huettel (2008) & 16 & $x$ & & & $x$ & & & $x$ \\
\hline Bach et al. (2009) & 2 & & $\mathrm{x}$ & & $x$ & & & $x$ \\
\hline Blackwood et al. (2004) & 6 & $x$ & & & $x$ & & & $x$ \\
\hline Elliott et al. (1999) & 12 & $x$ & & & $x$ & & & $x$ \\
\hline $\begin{array}{l}\text { Engelmann and Tamir } \\
\qquad(2009)\end{array}$ & 32 & $x(25)^{a}$ & $x(7)^{a}$ & $x$ & & $x$ & & \\
\hline Labudda et al. (2008) & 6 & $x$ & & & $x$ & & $x$ & \\
\hline Lee et al. (2008) & 3 & $x$ & & & $x$ & & $x$ & \\
\hline van Leijenhorst et al. (2006) & 16 & $x$ & & & $x$ & $x$ & & \\
\hline Xue et al. (2009) & 3 & $x$ & & $x$ & & & $x$ & \\
\hline Christopoulos et al. (2009) & 2 & $x$ & & & $x$ & $x$ & & \\
\hline Mohr et al. (2010a) & 2 & $x$ & & $x$ & & & $x$ & \\
\hline Number of foci & 232 & 172 & 60 & 131 & 101 & 101 & 92 & 67 \\
\hline
\end{tabular}

DR, Decision risk; AR, anticipation risk; Corr, correlation; Contr, contrast; $G$, gains; $G+L$, gains and losses; 0 , other. ${ }^{a}$ Engelmann and Tamir (2009) investigated risk in both contexts.

relationship. Because of this difference in research questions, new metaanalytic techniques were developed, which are adapted to the format of fMRI results. Specifically, whereas the key results of behavioral studies are test statistics ( $p, t$, or $z$ score) and effect sizes, test statistics in fMRI studies usually have meaning only together with information about the location of the effect, often given by the voxel with the highest test statistic. One frequently used meta-analytic technique that exploits this location information is ALE.

ALE is a quantitative meta-analysis technique that compares activation likelihoods calculated from observed activation foci with a null distribution of randomly generated activation likelihoods. In ALE, peak activation coordinates from a number of studies investigating similar effects are pooled (Chein et al., 2002; Turkeltaub et al., 2002; Laird et al., 2005). These coordinates are generally published relative to Talairach or Montreal Neurological Institute (MNI) space and therefore need to be spatially renormalized to a single template.

The resulting coordinates are used to generate "activation likelihoods" for each voxel in the brain. For each focus, ALE scores each voxel as a function of its distance from that focus using a three-dimensional Gaussian probability density function centered at the coordinates of the focus. As a result, ALE gets vectors of values for each voxel representing probabilities to belong to specific foci. These values are assumed to be independent (the existence of one focus does not give information about whether another focus will occur) and are combined with the addition rule for log-probabilities, yielding so-called ALE statistics. The ALE statistic represents the probability of a certain voxel to belong to any of the included foci.

To test for significance, the ALE statistic in each voxel is compared with a null distribution, generated via repeatedly calculating ALE statistics from randomly placed activation foci (same number as included in the study). This null distribution is then used to estimate the threshold 


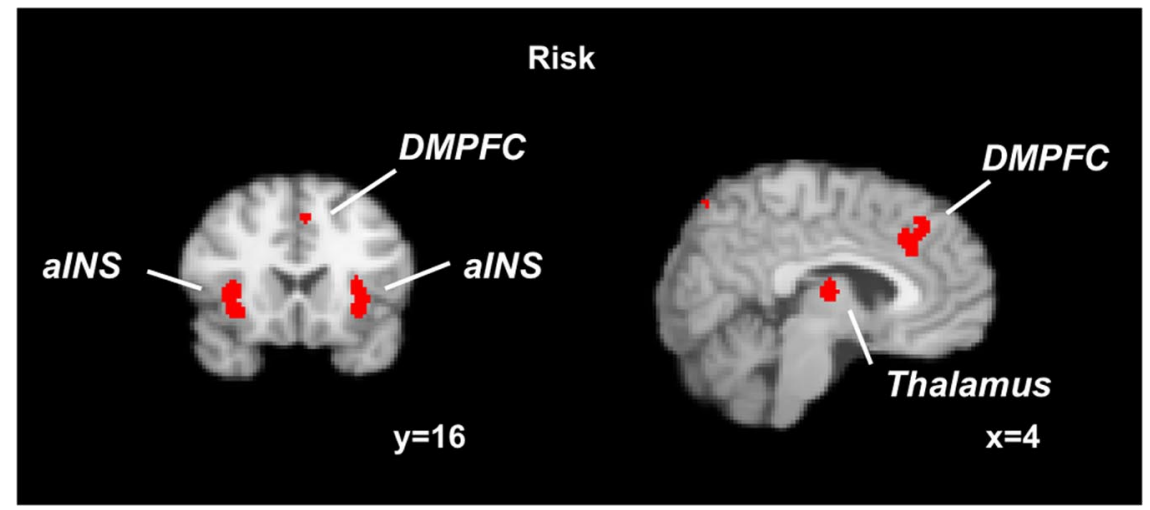

Figure 1. Neural representations of risk. Results from an ALE meta-analysis on risk independent of the context (decision risk or anticipation risk) and the domain (gains + losses or only gains in which risk was investigated). Activated clusters included bilateral aINS, DMPFC, and thalamus (FDR of $<0.05$; cluster size of $>200 \mathrm{~mm}^{3}$ ).

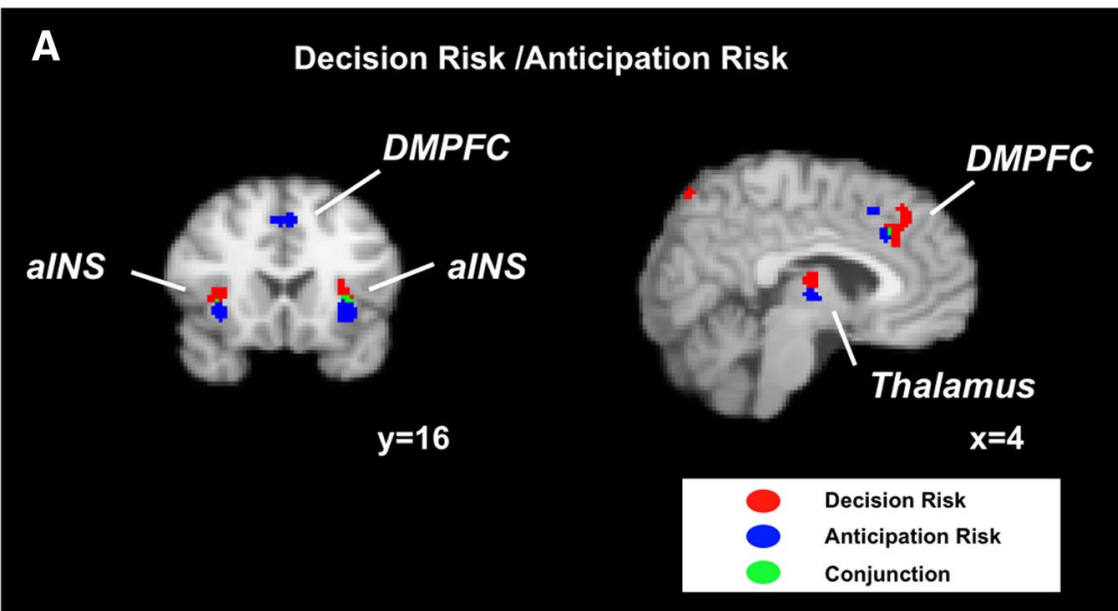

B Decision Risk vs. Anticipation Risk
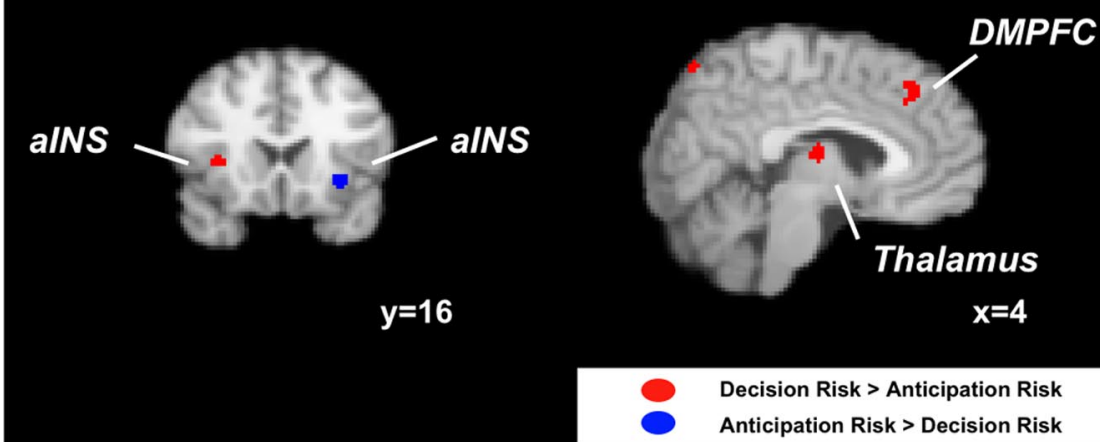

Decision Risk > Anticipation Risk

Anticipation Risk > Decision Risk

Figure 2. Neural representations of decision risk and anticipation risk. $\boldsymbol{A}$, Risk activated bilateral alNS and DMPFC in both a meta-analysis on decision risk and a meta-analysis on anticipation risk (although there is in all cases only a small overlap). Right DLPFC and right parietal cortex (both not displayed) were solely activated by decision risk. B, Right alNS, right DLPFC (not displayed), DMPFC, and right parietal cortex (not displayed) were more likely to be activated by decision risk compared with anticipation risk in a meta-analysis on the contrast between the two contexts in which risk was investigated. Left alNS, in contrast, was more likely to be activated by anticipation risk.

resulting for a given false discovery rate (FDR). Finally, a cluster threshold (minimum spatial extent of significant clusters) can be applied.

The ALE meta-analysis can also be used to contrast two independent meta-analyses. In this case, the ALE statistic in each voxel is calculated as the difference in ALE values between the two meta-analyses. Whereas
ALE values can only be positive in a single ALE meta-analysis, they can be negative as well in a contrast of ALE meta-analyses. The null distribution is calculated accordingly via calculating the differences between ALE statistics of randomly generated foci (again, same number of foci as included in the respective studies). Additional steps of the analysis equal the procedure used for a single ALE meta-analysis described above.

The ALE procedure was recently improved in that activation foci are now smoothed only in gray matter and in that group statistics are calculated as random effects, so that results can now be generalized beyond the studies in the sample (Eickhoff, 2009). Because this approach does not yet allow contrasting two sets of foci, we applied the standard approach in this study. Still, we report the results of each single meta-analysis using the new approach in the supplemental data (available at www. jneurosci.org as supplemental material) and show that they do not differ qualitatively from the results obtained with the standard approach. Actually, most clusters of significant foci were larger using the new approach (see supplemental data, available at www.jneurosci. org as supplemental material).

Application of the ALE meta-analysis. We first transformed MNI coordinates into Talairach space using the icbm2tal (Lancaster et al., 2007) transformation implemented in the GingerALE toolbox (http://brainmap.org; Research Imaging Center of the University of Texas Health Science Center, San Antonio, Texas). Second, we calculated different ALE meta-analyses with GingerALE. The ALE statistics in each of these meta-analyses is calculated by modeling each equally weighted activation peak using a three-dimensional Gaussian probability density function with a full-width half-maximum that is calculated as the average smoothing kernel of the included studies weighted with the number of foci for each study. To test for significance, we generated null distributions from 10,000 permutations for each meta-analysis.

The first meta-analysis included all riskrelated foci (risk analysis), independent of the context. Additionally, we divided risk-related foci in two groups according to their context (decision risk vs anticipation risk) and conducted separate meta-analyses for each group of foci (decision risk analysis and anticipation risk analysis). A contrast of these two metaanalyses identified regions that are more active in one context than in the other context (decision risk vs anticipation risk analysis). Similarly, we divided risk-related foci into two different groups according to the presence of losses (gains only and gains + losses, respectively). A third group of foci that represented neither gains nor gains + losses (but, e.g., right vs wrong or pain) was omitted from these analyses. To identify the effect of losses on risk processing, we contrasted the gains foci with the gains + losses foci (gains + losses vs gains only analysis). One has to note, however, that the two comparisons of groups of foci (decision risk vs anticipation risk and gains + losses vs gains, respectively) were not independent 
(Table 1). Most foci from the gains group were investigated during decision making (and vice versa), whereas most foci from the gains + losses group also belong to the set of foci for anticipation risk.

\section{Results}

In the risk analysis, we included all riskrelated foci from all studies (232 foci) independent of the specific context (decision context vs anticipation context) and domain (gains + losses vs gains). We found representations of risk in bilateral anterior insula (aINS), thalamus, dorsomedial prefrontal cortex (DMPFC), right dorsolateral prefrontal cortex (DLPFC), right parietal cortex, left precentral gyrus, and occipital cortex (supplemental Table S2, available at www.jneurosci.org as supplemental material) (Fig. 1).

To analyze the differences in representation of decision risk and anticipation risk, we conducted separate meta-analyses of studies involving these types of risk. The goal was to identify common neural substrates by using a conjunction analysis and to identify dissociations by contrasting the results of the meta-analyses. Both decision risk and anticipation risk activated bilateral aINS, DMPFC, and thalamus (supplemental Table S3, S4, available at www.jneurosci.org as supplemental material) (Fig. 2A). Significant clusters of the two contexts showed, however, only small overlap in these regions or did not overlap at all (thalamus).

To investigate activations associated only with decision or anticipation risk, respectively, we conducted an ALE metaanalysis on the contrast between decision risk and anticipation risk (decision risk vs anticipation risk analysis). Right aINS, DMPFC, DLPFC, parietal cortex, striatum, and occipital cortex were significantly more likely to be activated by decision risk, whereas left aINS and left superior temporal gyrus (STG) showed significantly higher ALE values for anticipation risk (supplemental Table S5, available at www.jneurosci.org as supplemental material) (Fig. 2B).

Because tasks that investigated neural representations of risk sometimes only included gains as possible outcomes and others also included losses, we formed two groups of foci according to this criterion. The goal of these analyses was to investigate whether risk is processed differently if subjects could lose money. Here we again conducted separate meta-analyses for the two groups of foci to identify common neural substrates and contrasted the results of the meta-analyses to identify significant differences. We found risk-related activations in both analyses in right aINS, DMPFC, and thalamus (supplemental Tables S6, S7, available at www.jneurosci.org as supplemental material) (Fig. $3 A$ ). Only the cluster in right aINS, however, showed an overlap between the two analyses. When contrasting the two sets of foci, we found that left aINS, left STG, and left precentral gyrus were more likely to be activated when losses were possible, whereas DMPFC, DLPFC, right parietal cortex, thalamus, and occipital cortex were more likely to be activated if only gains were possible (supplemental Table S8, available at www.jneurosci.org as supplemental material) (Fig. 3B).

\section{Discussion}

In our everyday life, we often have to make decisions with risky consequences. In fact, the outcome of a specific action is rarely certain. That is why risk has become an important concept in decision sciences. In the present study, we investigated three main questions regarding risk processing, namely (1) how risk processing is influenced by emotions, (2) whether the neural processing of risk is context-dependent, differing between decision making and pure outcome anticipation, and (3) whether risk is processed differently in the brain when individuals are faced with potential losses.

To answer these questions, we conducted quantitative coordinate-based meta-analyses on studies investigating neural representations of risk. By using the ALE approach (Laird et al., 2005), we can base our argumentation not only on a single study or a qualitative view on several studies but on a quantitative integration of 30 studies investigating risk. Although the ALE approach does not take the cluster size and significance-level ( $p, t$, or $z$ score) of an activated cluster into account, it offers the opportunity to locate an effect precisely, which is especially impor- 


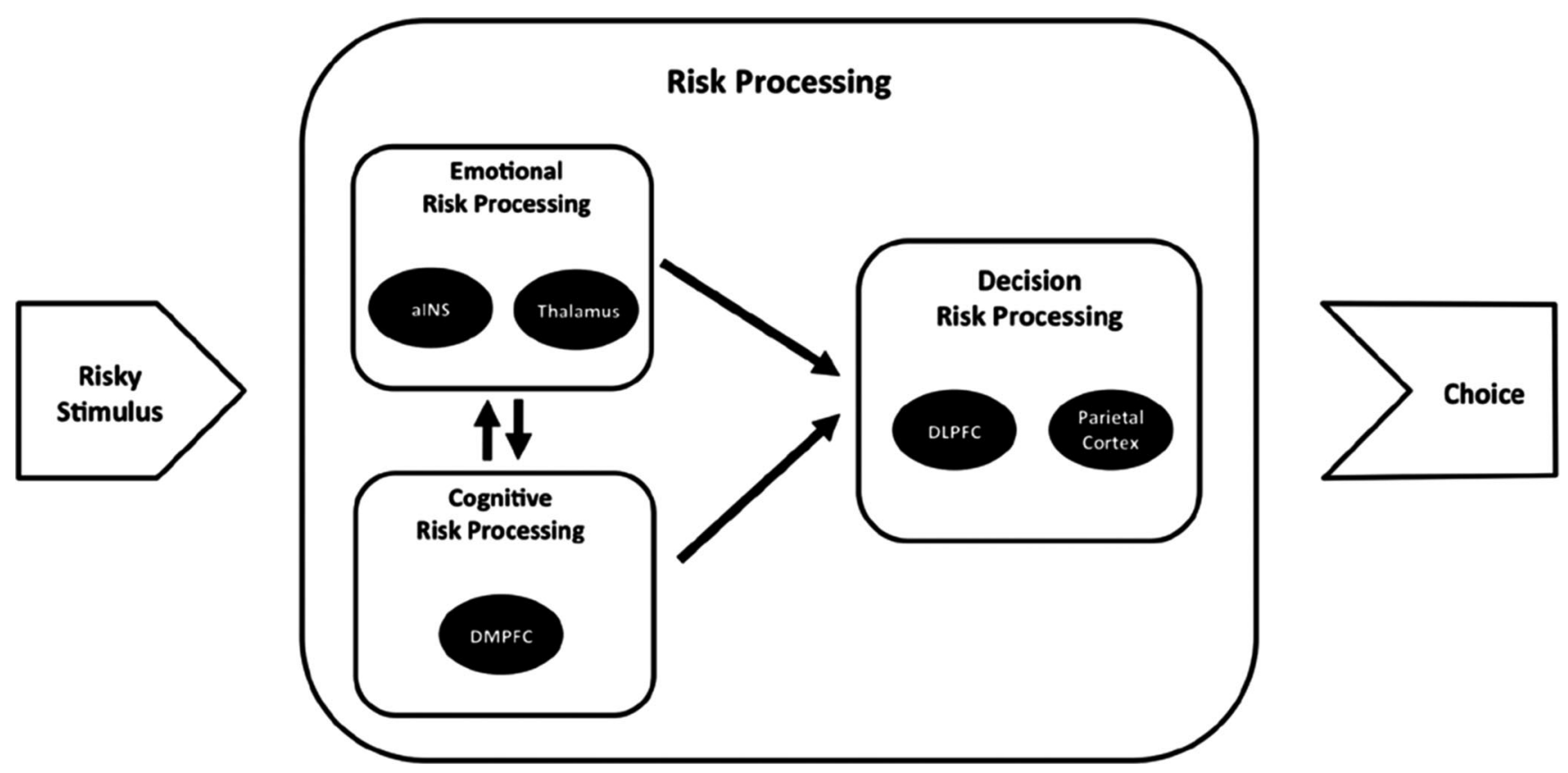

Figure 4. A potential mechanism of risky decision making. A risky stimulus such as a gamble with uncertain outcomes or a choice menu with different financial investments is initially evaluated on an emotional level. Activity in the alNS thereby serves as an estimate for the potential of the risky stimulus to result in an unwanted outcome, whereas the thalamus reflects important aspects of potential outcomes (e.g., their variability). At the cognitive level, the risky stimulus is processed in the DMPFC. Both parts of risk processing (emotional and cognitive) inform the actual decision process performed in DLPFC and parietal cortex. In situations, in which no decision has to be made, such as in the bingo game, the process concludes after emotional and cognitive risk processing.

tant in the context of large brain regions that likely consist of subregions serving different functions (e.g., medial prefrontal cortex) (Venkatraman et al., 2009a). Note that an alternative approach for fMRI meta-analyses could summarize studies based on effect sizes (and their SEs). This approach would have the advantage that small effects that remained undetected in single studies could be discovered as being significant. To our knowledge, however, no fMRI meta-analysis based on effect sizes has been published to date. One reason for this is that fMRI metaanalyses are only feasible, when whole brain maps of these statistics are available. When effect sizes are reported only for significant activations (as is currently the standard), effect sizes for all other areas remain unknown, and there is currently no method to estimate them with any confidence (Costafreda, 2009). Hence, the current reporting format of fMRI studies does not allow performing (effect-size based) meta-analyses that could detect weak but stable effects that were not published because of a publication bias for results with strong effects (that is inherent to fMRI studies with often small sample sizes). Note, however, that our identification of the thalamus as an important part of the risk processing network shows that coordinate-based meta-analyses allow identifying task-relevant brain regions that are activated in many studies and yet have remained unnoticed before.

We identified a network including bilateral aINS, dorsomedial thalamus, posterior thalamus, DMPFC, right DLPFC, and right parietal cortex for processing risk. The aINS was active for both decision risk and anticipation risk but predominantly when individuals were faced with potential losses. The aINS is regarded as a key brain region in emotion processing and arousal (Quartz, 2009) but also in the mapping of internal bodily states (Critchley, 2005; Craig, 2009). Several studies related activity in the aINS especially to aversive emotions such as fear, sadness, disgust, or anxiety (for review, see Phan et al., 2002; Paulus and Stein, 2006). Thus, our results clearly support the hypothesis that aversive emotions are implicated in risk processing independent of the context but predominantly (not solely) when individuals are faced with potential losses.

Standing alone, this conclusion is based on reverse inference (Poldrack, 2006) and needs to be qualified because the aINS is also active in a variety of tasks not explicitly related to emotions (Yang et al., 2010). Still, additional evidence comes from behavioral research on the influence of emotions on decision making. Several studies demonstrated that incidental emotions (i.e., emotions not related to the decision problem) significantly influence judgment and decision making. Risk judgments, for example, change when individuals perceive fear or anger (Lerner and Keltner, 2000).

The thalamus is one of the most ignored brain regions in functional neuroimaging. Although most of the studies included in our meta-analyses found risk representations in the thalamus, none of the studies discussed them. We found representations of risk in posterior and dorsomedial thalamus. The posterior thalamus was found to be active in the processing of emotions such as regret (Chandrasekhar et al., 2008) and showed stronger activity for losses compared with gains (Xu et al., 2009). Furthermore, the posterior thalamus is connected to the parietal cortex, which we also found to be active in risk processing. The dorsomedial thalamus is part of the striatal loop and reflects information about reward magnitudes (Glimcher and Lau, 2005). Interestingly, activity in the dorsomedial thalamus covaries with the magnitude of the smaller of two possible rewards (Minamimoto et al., 2009). The dorsomedial thalamus is also connected with the prefrontal cortex, a region that is consistently implicated in risk processing. Thus, both parts of the thalamus likely process important aspects of the risky stimulus and relay them to other brain regions of the risk processing network.

The DMPFC was active during decision risk and anticipation risk as well as in both domains (gains + losses and gains). It was, however, more likely to be active during decision risk compared with anticipation risk. Activity in the DMPFC was found in a 
variety of different tasks related to the cognitive processing of stimuli. These studies included investigations of response conflict, error monitoring, decision making, as well as strategy selection (for review, see Ridderinkhof et al., 2004; Venkatraman et al., $2009 \mathrm{~b})$. As recently reported, some of these functions can be attributed to subregions of the DMPFC (Venkatraman et al., 2009a). The cluster that we identified overlaps with the subregions for decision control and strategy control in the study by Venkatraman et al. (2009a). Because taking risks can be seen as acting against the dominant strategy of avoiding risks in riskaverse individuals, our findings are consistent with those previous findings about neural representations of strategy control.

We further found that both the right DLPFC and parietal cortex were active for decision risk but not for anticipation risk. Both brain regions were usually observed in the context of decision making (for review, see Heekeren et al., 2008). In the context of reward-based decision making, activity in the right DLPFC is related to valuing choice options (Camus et al., 2009; Mohr et al., 2010a). These results indicate that DLPFC and parietal cortex do not play a general role in risk processing but a specific role in risk processing during decision making.

In summary, our finding of insula activity supports the hypothesis that emotions are implicated in risk processing. The differential activation patterns for decision risk versus anticipation risk and for the gain versus gain + loss domain suggest that risk processing on the neural level is context dependent and specifically influenced by potential losses (note, however, that context and domain are not fully independent in the fMRI studies we found).

Based on the results of our meta-analyses, we propose the following account of a risk-processing mechanism (Fig. 4): when individuals observe a risky stimulus such as a gamble with uncertain outcomes or an investment option, two parallel and reciprocal risk processes are induced, an emotional and a cognitive risk process. On the emotional level, activity in the aINS initially serves as a fast and rough estimate for the potential of the stimulus to result in an unwanted outcome (e.g., a loss). At the same time, this signal prepares the organism to take action to avoid the unwanted outcome. Activity in the thalamus could thereby reflect an anticipation of regret in response to possible outcomes of the risky stimulus. The DMPFC evaluates the risk of the stimulus on a cognitive level, for instance, computing the variance of outcomes or the probability of a loss, thereby using the information from the aINS and the thalamus as a first estimate for the riskiness of the stimulus. During this process, information is repeatedly exchanged between DMPFC on the one hand and aINS and thalamus on the other hand, updating the emotional response to the stimulus, which in turn informs the cognitive processing of risk. If a decision has to be made, parietal cortex and DLPFC integrate the risk information with other aspects of the stimulus (e.g., expected reward) and form the final decision. If no decision has to be made, like in the bingo game, the process stops after risk processing on the emotional and the cognitive level. The mechanism proposed here is compatible with the general approach of the risk-as-feelings hypothesis (Loewenstein et al., 2001). Note that the temporal sequence of risk processing we propose cannot be derived from our meta-analysis or fMRI data in general. EEG and/or magnetoencephalographic experiments could shed light on the temporal sequence of cortical risk processing. Moreover, the suggested mechanism is based on results of experiments that investigated risk processing in the presence of only gains or both gains and losses. No study has so far investigated risk processing specifically in the loss domain. Future research should target this obvious lack of research and complete the picture of risk processing for the loss domain.

\section{References}

Bach DR, Seymour B, Dolan RJ (2009) Neural activity associated with the passive prediction of ambiguity and risk for aversive events. J Neurosci 29:1648-1656.

Behrens TE, Woolrich MW, Walton ME, Rushworth MF (2007) Learning the value of information in an uncertain world. Nat Neurosci 10:1214-1221.

Blackwood N, Ffytche D, Simmons A, Bentall R, Murray R, Howard R (2004) The cerebellum and decision making under uncertainty. Brain Res Cogn Brain Res 20:46-53.

Bossaerts P, Preuschoff K, Hsu M (2009) The neurobiological foundations of valuation in human decision making under uncertainty. In: Neuroeconomics. Decision making and the brain (Glimcher PW, Camerer CF, Fehr E, Poldrack RA, eds), pp 353-366. London: Elsevier.

Cabeza R, Nyberg L, Park DC, eds (2005) Cognitive neuroscience of aging. New York: Oxford UP.

Camus M, Halelamien N, Plassmann H, Shimojo S, O’Doherty J, Camerer C, Rangel A (2009) Repetitive transcranial magnetic stimulation over the right dorsolateral prefrontal cortex decreases valuations during food choices. Eur J Neurosci 30:1980-1988.

Chandrasekhar PV, Capra CM, Moore S, Noussair C, Berns GS (2008) Neurobiological regret and rejoice functions for aversive outcomes. Neuroimage 39:1472-1484.

Chein JM, Fissell K, Jacobs S, Fiez JA (2002) Functional heterogeneity within Broca's area during verbal working memory. Physiol Behav 77:635-639.

Christopoulos GI, Tobler PN, Bossaerts P, Dolan RJ, Schultz W (2009) Neural correlates of value, risk, and risk aversion contributing to decision making under risk. J Neurosci 29:12574-12583.

Costafreda SG (2009) Pooling FMRI data: meta-analysis, mega-analysis and multi-center studies. Front Neuroinformatics 3:33.

Craig AD (2009) How do you feel-now? The anterior insula and human awareness. Nat Rev Neurosci 10:59-70.

Critchley HD (2005) Neural mechanisms of autonomic, affective, and cognitive integration. J Comp Neurol 493:154-166.

Critchley HD, Mathias CJ, Dolan RJ (2001) Neural activity in the human brain relating to uncertainty and arousal during anticipation. Neuron 29:537-545.

Damasio AR, Tranel D, Damasio H (1991) Somatic markers and guidance of behaviour:theory and preliminary testing. In: Frontal lobe function and dysfunction (Levin HS, Eisenberg HM, Benton AL, eds). New York: Oxford UP.

Dreher JC, Kohn P, Berman KF (2006) Neural coding of distinct statistical properties of reward information in humans. Cereb Cortex 16:561-573.

Eickhoff SB, Laird AR, Grefkes C, Wang LE, Ziles K, Fox PT (2009) Coordinate-based activation likelihood estimation meta-analysis of neuroimaging data: a random-effects approach based on empirical estimates of spatial uncertainty. Hum Brain Mapp 30:2907-2926.

Elliott R, Rees G, Dolan RJ (1999) Ventromedial prefrontal cortex mediates guessing. Neuropsychologia 37:403-411.

Engelmann JB, Tamir D (2009) Individual differences in risk preference predict neural responses during financial decision-making. Brain Res 1290:28-51.

Fox PT, Poldrack RA (2009) Prospect theory and the brain. In: Neuroeconomics. Decision making and the brain (Glimcher PW, Camerer CF, Fehr E, Poldrack RA, eds), pp 145-173. London: Elsevier.

Glimcher PW, Lau B (2005) Rethinking the thalamus. Nat Neurosci 8:983-984.

Grinband J, Hirsch J, Ferrera VP (2006) A neural representation of categorization uncertainty in the human brain. Neuron 49:757-763.

Heekeren HR, Marrett S, Ungerleider LG (2008) The neural systems that mediate human perceptual decision making. Nat Rev Neurosci 9:467-479.

Hsu M, Bhatt M, Adolphs R, Tranel D, Camerer CF (2005) Neural systems responding to degrees of uncertainty in human decision-making. Science 310:1680-1683.

Huettel SA (2006) Behavioral, but not reward, risk modulates activation of prefrontal, parietal, and insular cortices. Cogn Affect Behav Neurosci 6:141-151. 
Huettel SA, Song AW, McCarthy G (2005) Decisions under uncertainty: probabilistic context influences activation of prefrontal and parietal cortices. J Neurosci 25:3304-3311.

Knight FH (1921) Risk, uncertainty and profit. Boston: Houghton Mifflin.

Knutson B, Bossaerts P (2007) Neural antecedents of financial decisions. J Neurosci 27:8174-8177.

Kuhnen CM, Knutson B (2005) The neural basis of financial risk taking. Neuron 47:763-770.

Labudda K, Woermann FG, Mertens M, Pohlmann-Eden B, Markowitsch HJ, Brand M (2008) Neural correlates of decision making with explicit information about probabilities and incentives in elderly healthy subjects. Exp Brain Res 187:641-650.

Laird AR, Fox PM, Price CJ, Glahn DC, Uecker AM, Lancaster JL, Turkeltaub PE, Kochunov P, Fox PT (2005) ALE meta-analysis: controlling the false discovery rate and performing statistical contrasts. Hum Brain Mapp 25:155-164.

Lancaster JL, Tordesillas-Gutiérrez D, Martinez M, Salinas F, Evans A, Zilles K, Mazziotta JC, Fox PT (2007) Bias between MNI and Talairach coordinates analyzed using the ICBM-152 brain template. Hum Brain Mapp 28:1194-1205.

Lee TM, Leung AW, Fox PT, Gao JH, Chan CC (2008) Age-related differences in neural activities during risk taking as revealed by functional MRI. Soc Cogn Affect Neurosci 3:7-15.

Lerner JS, Keltner D (2000) Beyond valence: toward a model of emotionspecific influences on judgement and choice. Cogn Emot 14:473-493.

Loewenstein GF, Weber EU, Hsee CK, Welch N (2001) Risk as feelings. Psychol Bull 127:267-286.

Markowitz H (1952) Portfolio selection. J Finance 7:77-91.

Matthews SC, Simmons AN, Lane SD, Paulus MP (2004) Selective activation of the nucleus accumbens during risk-taking decision making. Neuroreport 15:2123-2127.

Minamimoto T, Hori Y, Kimura M (2009) Roles of the thalamic CM-PF complex-basal ganglia circuit in externally driven rebias of action. Brain Res Bull 78:75-79.

Mohr PN, Biele G, Krugel LK, Li SC, Heekeren HR (2010a) Neural foundations of risk-return trade-off in investment decisions. Neuroimage 49:2556-2563.

Mohr PN, Li SC, Heekeren HR (2010b) Neuroeconomics and aging: neuromodulation of economic decision making in old age. Neurosci Biobehav Rev 34:678-688.

Park DC, Polk TA, Park R, Minear M, Savage A, Smith MR (2004) Aging reduces neural specialization in ventral visual cortex. Proc Natl Acad Sci U S A 101:13091-13095.

Paulus MP, Stein MB (2006) An insular view of anxiety. Biol Psychiatry 60:383-387.

Paulus MP, Hozack N, Zauscher B, McDowell JE, Frank L, Brown GG, Braff DL (2001) Prefrontal, parietal, and temporal cortex networks underlie decision-making in the presence of uncertainty. Neuroimage 13:91-100.

Paulus MP, Rogalsky C, Simmons A, Feinstein JS, Stein MB (2003) Increased activation in the right insula during risk-taking decision making is related to harm avoidance and neuroticism. Neuroimage 19:1439-1448.

Phan KL, Wager T, Taylor SF, Liberzon I (2002) Functional neuroanatomy of emotion: a meta-analysis of emotion activation studies in PET and fMRI. Neuroimage 16:331-348.

Platt ML, Huettel SA (2008) Risky business: the neuroeconomics of decision making under uncertainty. Nat Neurosci 11:398-403.

Poldrack RA (2006) Can cognitive processes be inferred from neuroimaging data? Trends Cogn Sci 10:59-63.
Preuschoff K, Bossaerts P, Quartz SR (2006) Neural differentiation of expected reward and risk in human subcortical structures. Neuron 51:381-390.

Preuschoff K, Quartz SR, Bossaerts P (2008) Human insula activation reflects risk prediction errors as well as risk. J Neurosci 28:2745-2752.

Quartz SR (2009) Reason, emotion and decision-making: risk and reward computation with feeling. Trends Cogn Sci 13:209-215.

Rangel A, Camerer C, Montague PR (2008) A framework for studying the neurobiology of value-based decision making. Nat Rev Neurosci 9:545-556.

Ridderinkhof KR, Ullsperger M, Crone EA, Nieuwenhuis S (2004) The role of the medial frontal cortex in cognitive control. Science 306:443-447.

Rolls ET, McCabe C, Redoute J (2008) Expected value, reward outcome, and temporal difference error representations in a probabilistic decision task. Cereb Cortex 18:652-663.

Rushworth MF, Behrens TE (2008) Choice, uncertainty and value in prefrontal and cingulate cortex. Nat Neurosci 11:389-397.

Slovic P, Finucane ML, Peters E, MacGregor DG (2004) Risk as analysis and risk as feelings: some thoughts about affect, reason, risk, and rationality. Risk Anal 24:311-322.

Smith BW, Mitchell DG, Hardin MG, Jazbec S, Fridberg D, Blair RJ, Ernst M (2009) Neural substrates of reward magnitude, probability, and risk during a wheel of fortune decision-making task. Neuroimage 44:600-609.

Tobler PN, O'doherty JP, Dolan RJ, Schultz W (2006) Human neural learning depends on reward prediction errors in the blocking paradigm. J Neurophysiol 95:301-310.

Turkeltaub PE, Eden GF, Jones KM, Zeffiro TA (2002) Meta-analysis of the functional neuroanatomy of single-word reading: method and validation. Neuroimage 16:765-780.

van Leijenhorst L, Crone EA, Bunge SA (2006) Neural correlates of developmental differences in risk estimation and feedback processing. Neuropsychologia 44:2158-2170.

Venkatraman V, Rosati AG, Taren AA, Huettel SA (2009a) Resolving response, decision, and strategic control: evidence for a functional topography in dorsomedial prefrontal cortex. J Neurosci 29:13158-13164.

Venkatraman V, Payne JW, Bettman JR, Luce MF, Huettel SA (2009b) Separate neural mechanisms underlie choices and strategic preferences in risky decision making. Neuron 62:593-602.

Volz KG, Schubotz RI, von Cramon DY (2003) Predicting events of varying probability: uncertainty investigated by fMRI. Neuroimage 19:271-280.

Volz KG, Schubotz RI, von Cramon DY (2004) Why am I unsure? Internal and external attributions of uncertainty dissociated by fMRI. Neuroimage 21:848-857.

Weber BJ, Huettel SA (2008) The neural substrates of probabilistic and intertemporal decision making. Brain Res 1234:104-115.

Weber EU, Anderson CJ, Birnbaum MH (1992) A theory of perceived risk and attractiveness. Organ Behav Hum Decis Process 52:492-523.

Weber EU, Blais AR, Betz NE (2002) A domain-specific risk-attitude scale: Measuring risk perceptions and risk behaviors. J Behav Decis Mak 15:263-290.

Xu L, Liang ZY, Wang K, Li S, Jiang T (2009) Neural mechanism of intertemporal choice: from discounting future gains to future losses. Brain Res 1261:65-74.

Xue G, Lu Z, Levin IP, Weller JA, Li X, Bechara A (2009) Functional dissociations of risk and reward processing in the medial prefrontal cortex. Cereb Cortex 19:1019-1027.

Yang J, Weng X, Zang Y, Xu M, Xu X (2010) Sustained activity within the default mode network during an implicit memory task. Cortex 46:354366.

Yoshida W, Ishii S (2006) Resolution of uncertainty in prefrontal cortex. Neuron 50:781-789. 